\title{
Digitally Fabricated Mobile Spectrometer for Multipoint Continuous Spectroscopic Analysis of Light Environment in Greenhouse Tomato Canopies
}

\author{
Takehiko Hoshi $^{1}$, Kenta UedA ${ }^{2}$, Yoshihiro TAKIKAwA ${ }^{3}$ and Takaya AzUmA ${ }^{4}$ \\ ${ }^{1}$ Faculty of Biology-Oriented Science and Technology, Kindai University, 930 Nishimitani, Kinokawa, Wakayama 649-6493, Japan \\ ${ }^{2}$ Graduate School of Biology-Oriented Science and Technology, Kindai University, 930 Nishimitani, Kinokawa, \\ Wakayama 649-6493, Japan \\ ${ }^{3}$ Plant center, Institute of Advanced Technology, Kindai University, 14-1 Minamiakasaka, Kainan, Wakayama 642-0017, Japan \\ ${ }^{4}$ Wakayama Agricultural Experiment Station, 160 Takao, Kishigawa-cho, Kinokawa, Wakayama 640-0423, Japan
}

(Received September 20, 2017; Accepted May 2, 2018)

\begin{abstract}
A low-cost spectrometer, featuring a micro electro mechanical systems (MEMS) sensor, was designed and manufactured using digital fabrication techniques. The spectrometer was morphed into a zelkova leaf-shaped case using a three-dimensional printer. The control software, written in the Arduino sketch programing language, measured the flux of photons in the 400-800 $\mathrm{nm}$ range, at $1 \mathrm{~nm}$ resolution, every $5 \mathrm{~min}$. The obtained data were saved on a micro SD memory card in the comma separated values format. The spectrometer continuously operated for more than $3 \mathrm{~d}$ using a type B universal serial bus micro connector and a $10 \mathrm{Ah}$ mobile battery. Four manufactured spectrometers were installed inside and outside of a tomato canopy in the experimental greenhouse of Wakayama prefecture, and continuously measured the spectroscopic flux of photons for nine $\mathrm{d}$. The quantitative changes in the phytochrome photoequilibrium in the tomato leaves were estimated using the data from the continuous spectroscopic analysis. The effect of pruning under leaves on the illumination in the tomato canopy was quantified in terms of the leaf area index. We expect that the proposed low-cost and high-mobility spectrometer will be used in plant production fields.
\end{abstract}

Keywords: FabLab, micro-spectrometer C12880MA, multi-point simultaneous measurement, protected horticulture, spectroscopic measurement

\section{INTRODUCTION}

Accurate measurements and control of light environment can significantly affect plant growth; thus, light measurement and control techniques are important for increasing plant yield. The lowest-cost option amounts to measuring the illuminance, for which instruments are readily available; more advanced techniques address measurements of radiation and photon fluxes. For the evaluation of photosynthesis under various light sources, the most informative quantity is the flux of photosynthetic photons in the 400-700 nm range of wavelengths (Tibbitts et al., 1983). To comprehensively evaluate the light environment including photomorphogenesis, spectroscopic measurements and analysis are becoming indispensable. Many spectrometers have been introduced into plant research laboratories, leading to some significant advances (Kendrick and Kronenberg, 1986). However, because spectrometers are much more expensive than illuminance meters, this approach is not practical for use in small-scale fields, such as the ones that are cultivated by many farmers in Japan. Consequently, these relatively expensive devices remain unpopular.

The development of the micro electro mechanical systems (MEMS) technology has contributed to miniaturiza- tion and cost reduction of sensors (Kaajakari, 2009). Some micro-spectrometer MEMS devices that combine reflective concave brazed gratings and complementary metaloxide-semiconductor (CMOS) linear image sensors have been commercialized (Hamamatsu Photonics, 2017). To fabricate a measuring instrument that uses the MEMS technology, a computer system for controlling and a case for housing are required. Up to date, it has been difficult to solve these problems in research laboratories, and the existing prototypes have been bulky and costly. Recently, digital fabrication technology has been proposed for overcoming these technical problems in a small lot production (Walter-Herrmann and Büching, 2014). Based on the increasing use of open source hardware (computers) and three-dimensional (3D) printers, a framework for developing and manufacturing equipment for digital gadgets, called FabLab, was established (Pearce, 2012). Owing to this technology, fabrication of prototypes has become much less expensive.

A low-cost spectrometer, featuring an MEMS device and open source hardware, was designed and manufactured using a 3D printer. Four manufactured spectrometers were installed inside and outside of a tomato canopy in an experimental greenhouse, and were tested to determine their usability for continuous measurements and spectroscopic

Corresponding author: Takehiko Hoshi, fax: +81-736-77-4754, e-mail: hoshi@waka.kindai.ac.jp 


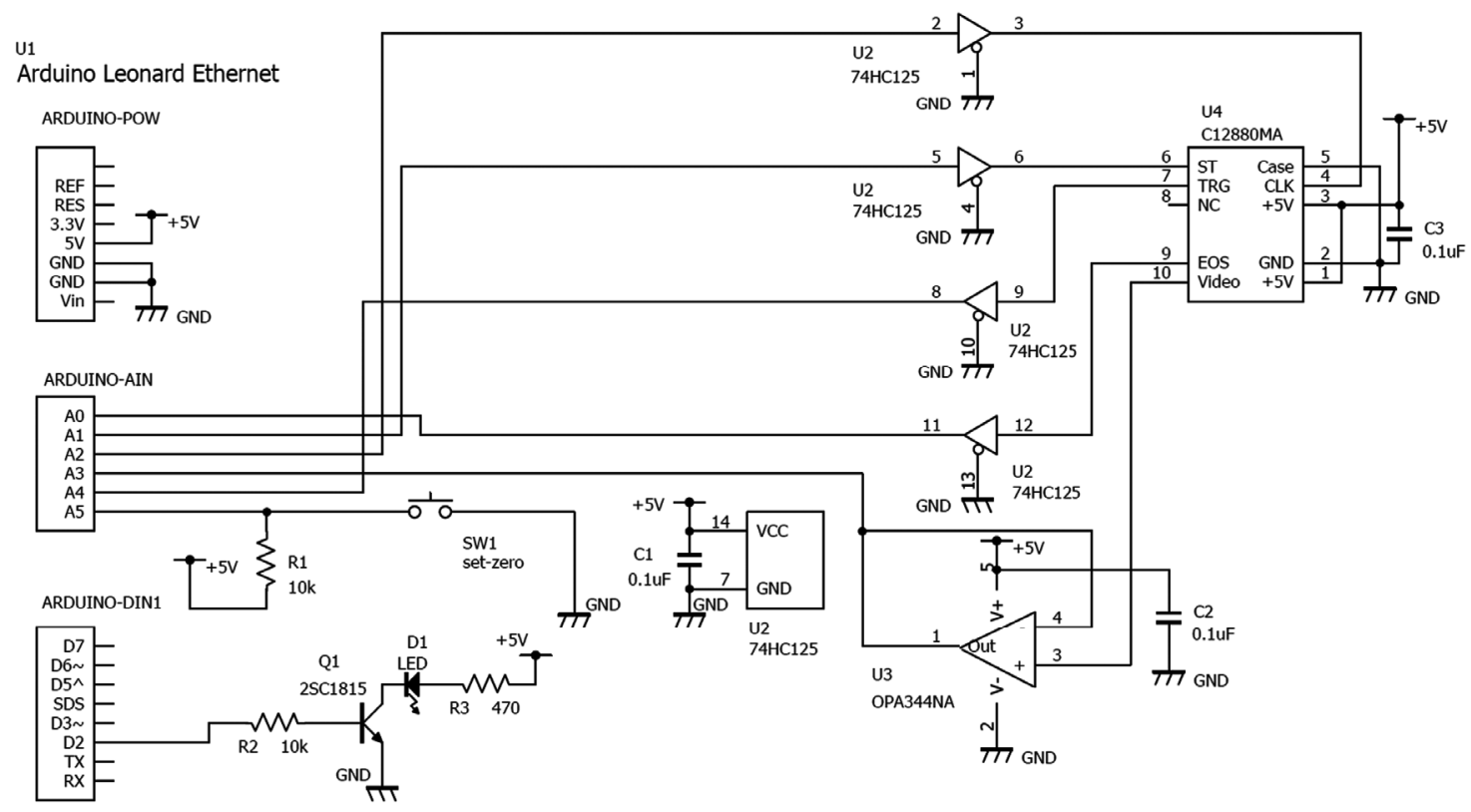

Fig. 1 Circuit diagram of the mobile spectrometer.

evaluation of the illumination environment.

\section{MATERIALS AND METHODS}

\section{MEMS device of micro-spectrometer}

A micro-spectrometer device, C12880MA (Hamamatsu Photonics, 2017) was utilized for prototyping the proposed mobile spectrometer. This fingertip-sized MEMS device was enclosed in a moisture proof metal hermetic package (volume, $2.65 \mathrm{~cm}^{3}$; weight, $5 \mathrm{~g}$ ). The light, incident through a slit, was spectrally separated by a reflective concave blazed grating, and photometry was performed using a 288 pixel CMOS line sensor. The wavelength sensitivity range was $340 \mathrm{~nm}$ to $850 \mathrm{~nm}$. The spectral resolution of the data sheet was lower than $15 \mathrm{~nm}$; however, that of the 15 devices that were used for prototyping was actually $\sim 10 \mathrm{~nm}$. The photometric signal from each pixel was processed by interpolation, and photometric values for the resolution of $1 \mathrm{~nm}$ were obtained as a video signal. The frequency of the clock pulses that were necessary to control the device was in the $0.2-5 \mathrm{MHz}$ range.

Hardware configuration

The computer that controlled the mobile spectrometer featured open source hardware, Arduino Leonard Ethernet (CPU: ATmega32U4, Clock: $16 \mathrm{MHz}, \mathrm{ROM}: 32 \mathrm{kB}$, RAM: $2.5 \mathrm{kB}$, EEPROM: $1 \mathrm{kB}$, micro SD memory card reader built in) (http://www.arduino.org/). The circuit diagram of the mobile spectrometer is shown in Fig. 1. Each signal line was buffered to suppress measurement errors owing to the generation of heat by the MEMS device. A pushbutton switch for zero point adjustment and a light emitting diode (LED) for indicating operation were installed.

For commercial applications in plant production fields, mobile spectrometers should be user-friendly. Thus, the housing case was designed in the shape of a plant leaf. A JPG image of a zelkova leaf (Zelkova serrata (Thunb.)

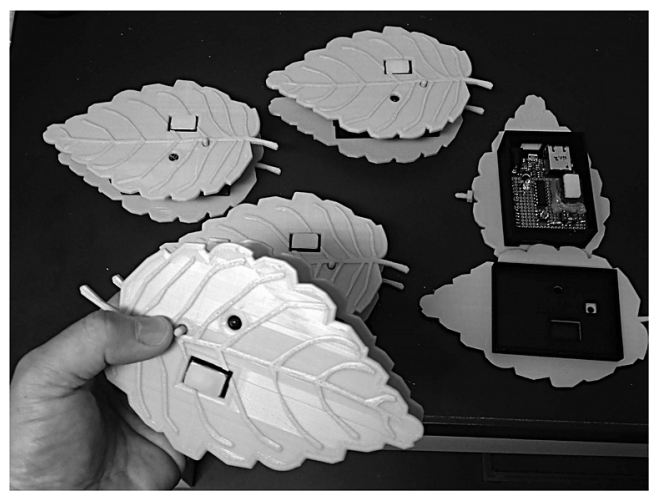

Fig. 2 Exterior and interior views of the mobile spectrometers fabricated using digital techniques.

Makino) was used for building a 3D model using a 3D builder (Microsoft Corp., Redmond, WA, USA). The size of the $3 \mathrm{D}$ model was adjusted and the model was further processed using 123D Design (Autodesk Inc., San Rafael, CA, USA) of CAD software; further, the model was rendered for printing using a 3D printer by SCOOVO Studio SE (Abee Corp., Yokohama, Japan). A housing case in the shape of a zelkova leaf was fabricated using a 3D printer (SCOOVO X9H, Abee Corp.), and fabrication of one leafshaped case required $\sim 100 \mathrm{~g}$ of polylactic acid resin. The case's dimensions were $171 \times 100 \times 36 \mathrm{~mm}^{3}$, and its overall weight was $144 \mathrm{~g}$. The manufactured mobile spectrometer is shown in Fig. 2. This spectrometer was branded "Zelko".

\section{Software development}

The control program was written in Arduino Sketch using Arduino IDE version 1.7.10. The first prototyping of the control software that only used Arduino Sketch's builtin functions was not satisfactory. The main reason was probably the fact that the clock pulse signal output to C12880MA through the digital output port was coded using the "digitalWrite()" built-in function. In the specifica- 
tion of $\mathrm{C} 12880 \mathrm{MA}$, the clock frequency is required to be $0.2 \mathrm{MHz}$ or more (cycle time is $5 \mu$ s or less). However, it took $5.15 \mu \mathrm{s}$ to execute the function, owing to overhead instructions. If the clock signal could be flipped by calling the function only (without overhead instructions), the cycle time of the pulse could be $10.3 \mu \mathrm{s}(0.097 \mathrm{MHz})$. Furthermore, when some components such as the analog to digital (A/D) conversion were added to the module that generated clock pulses, the allowable range of the specification of C12880MA increased significantly, and the device became dysfunctional. To overcome this problem, by manipulating the ATmega32U4 register directly to control the signal of the output port, time required to generate the clock pulse was $160 \mathrm{~ns}$ ( $\sim 32$ times faster). The frequency division ratio of the A/D conversion clock was also changed, and the conversion cycle time improved from $112 \mu$ s to $19 \mu \mathrm{s}$. When the measured data were read from C12880MA, an A/D converter was required. In this case only, the cycle time of the clock increased more than six-fold compared with the specification; however, the spectrometer functioned normally. As a result of these modifications, the control software could be operated only using the Arduino Leonard Ethernet board with ATmega32U4.

A flowchart of the control software is schematically shown in Fig. 3. After starting "Zelko", the user covers the light receptor, to compensate for the offset owing to the background noise of the CMOS line sensor. The first spectral measurement is performed $4 \mathrm{~s}$ later, and the measured data are written in the comma separated values (CSV) format to a micro SD memory card. Thereafter, measurements and data writing are performed every $5 \mathrm{~min}$. The program module of the control software (ROM) requires 20,180 bytes, while variables (RAM) require 1,804 bytes. The source code and the shape data (for a $3 \mathrm{D}$ printer) will be available for downloading.

Optical adjustments

A milky white acrylic board (thickness, $3 \mathrm{~mm}$ ) was at-

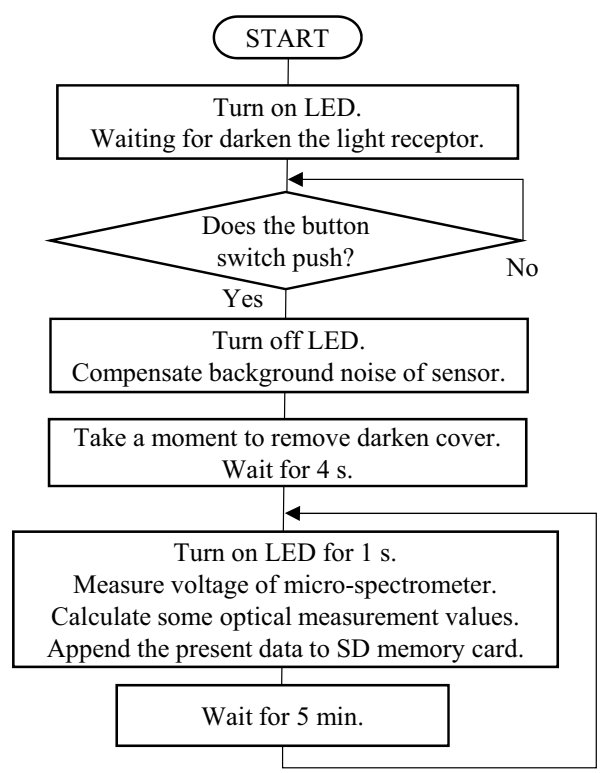

Fig. 3 Schematic flowchart of the control program. tached to the light receptor for diffusing the direct sunlight. Since ultraviolet (UV) light cut acrylic resin was used, transmission of light with wavelengths under $400 \mathrm{~nm}$ was significantly attenuated. Therefore, the wavelength of 400 $\mathrm{nm}$ was used as the shortest measured wavelength.

The video output for C12880MA was in the units of voltage (saturation output voltage, $4.3 \mathrm{~V} \mathrm{~nm}^{-1}$ ). A reference spectrometer, Flame-S\#3 (Ocean Optics Inc., Largo, FL, USA), calibrated using a NIST-compliant halogen light source (HL-3P-CAL, Ocean Optics Inc.), was employed for converting the voltage into the spectral radiation flux $\left(\mu \mathrm{W} \mathrm{m} \mathrm{mm}^{-1}\right)$. Since the measurement stability of the reference spectrometer was compromised for wavelengths above $800 \mathrm{~nm}$, the measurement limit on longer wavelengths was set to $800 \mathrm{~nm}$. In the $400-800 \mathrm{~nm}$ range, a fourth-order conversion formula (coefficient of determination $\left(\mathrm{R}^{2}\right)=0.9523$ ) was obtained.

Conversion from the radiation flux units to photon flux units utilized equation (1):

$$
E_{\lambda}=h \frac{C}{\lambda}
$$

Here, $h$ is the Planck constant $\left(6.62607 \times 10^{-34} \mathrm{~J} \mathrm{~s}\right), c$ is the speed of light in vacuum $\left(2.99792 \times 10^{8} \mathrm{~m} \mathrm{~s}^{-1}\right), \lambda$ is the wavelength of light (m), and $E_{\lambda}$ is the energy at $\lambda$ per 1 mol of photons $(\mathrm{J})$.

Then, the photon flux at $\lambda$ per $1 \mathrm{~nm}$ width $\left(P F_{\lambda}\right.$, mol $\mathrm{m}^{-2} \mathrm{~s}^{-1} \mathrm{~nm}^{-1}$ ) can be obtained from equation (2) using the Avogadro number $\left(\mathrm{N}_{\mathrm{A}}, 6.02214 \times 10^{23} \mathrm{~mol}^{-1}\right)$ and the radiation flux at $\lambda$ per $1 \mathrm{~nm}$ width $\left(R F_{\lambda}, \mathrm{W} \mathrm{m}^{-2} \mathrm{~nm}^{-1}\right)$ :

$$
P F_{\lambda}=\frac{R F_{\lambda} \cdot \lambda}{\mathrm{h} \cdot \mathrm{C} \cdot \mathrm{N}_{\mathrm{A}}}=8.35936 R F_{\lambda} \cdot \lambda
$$

"Zelko" measured $P F_{\lambda}$ by iteratively changing the accumulation time of the charge coupled device (CCD) line sensor up to 46.7-fold of the minimal accumulation time, in 7 steps. Then, the measured value with the longest photon storage time that did not reach the saturation output voltage was adopted. The dynamic range at $532 \mathrm{~nm}$ was measured using a green laser diode.

Finally, $P F_{\lambda}$ values were measured for the $400 \mathrm{~nm}$ to $800 \mathrm{~nm}$ range, in steps of $1 \mathrm{~nm}$, using "Zelko". The photosynthetic photon flux (PPF: $400 \mathrm{~nm}$ to $700 \mathrm{~nm}$ ), the blue photon flux (BPF: $400 \mathrm{~nm}$ to $499 \mathrm{~nm}$ ), the green photon flux $(G P F$ : $500 \mathrm{~nm}$ to $599 \mathrm{~nm})$, the red photon flux $(R P F$ : $600 \mathrm{~nm}$ to $699 \mathrm{~nm}$ ), and the far-red photon flux (FRPF: $700 \mathrm{~nm}$ to $800 \mathrm{~nm}$ ) were also calculated by accumulating the specified wavelength range of $P F_{\lambda}$ values.

The simultaneous measured $P P F$ values of 5 sets of "Zelko" in the same natural sun light deviated from the mean value with ranged $-3.18 \%$ to $+2.46 \%$ (standard deviation: $2.02 \%)$. Therefore, coefficients for correcting this deviation were incorporated into the control software. To fabricate a prototype, the induced coefficient obtained from the measured values of the independent PPF meter should be provided to the control software.

Spectroscopic measurements in a greenhouse tomato canopy

Four "Zelko" spectrometers were installed inside and outside of a plant canopy in the experimental greenhouse No. 54 at the Wakayama Agricultural Experiment Station. 
The greenhouse is a single steel frame plastic house $(7 \times 28$ $\mathrm{m}^{2}$ ) covered with a polyolefin composite film, with a 1layer curtain installed. Tomatoes (Solanum lycopersicum L. ' $F_{1}$ Momotaro Peace') were planted in hydroponic beds in the greenhouse, in two rows. The inter-bed distance was $140 \mathrm{~cm}$, and the inter-plant distance was $35 \mathrm{~cm}$. The spectrometers were installed inside and outside the tomato canopy as shown in Fig. 4. The spectroscopic data collected by the four spectrometers corresponded to the shoot apex $(T)$, middle of the plant $(\mathrm{M})$, the bed surface $(B)$, and the aisle (A), according to the locations of the spectrometers. The measurements were performed from December 10 to 18,2016 . The lower leaves of tomatoes were pruned on December 14 , for controlling the leaf area.

Leaf area index (LAI) was calculated by dividing the total leaf area of the plant by its cultivated land area. The LAI quantitatively captures the luxuriant growth degree and the light receiving efficiency of the plant. Using the experimentally established fact that one tomato leaf transmits $\sim 60 \%$ of far-red light, LAI can be estimated from optical measurements using the Lambert-Beer law (Hoshi et al., 2014). The estimated LAI values were calculated by measuring two positions of FRPF and changes in the light absorption efficiency after pruning under leaves was evaluated.

Phytochrome is a primary chromoprotein photoreceptor controlling plant morphology. Phytochrome exists in two forms, $P r$, which absorbs red light, and in the physiologically active form, Pfr, that absorbs far-red light (Holmes and Smith, 1975). Switching from one form to another is achieved by the absorption of light. The light absorption wavelength bands of the two forms overlap, and when the light of a certain wavelength distribution is irradiated, the photostationary state changes instantaneously (Linschitz et al., 1966). The amount in the photoequilibrium state $(P E)$

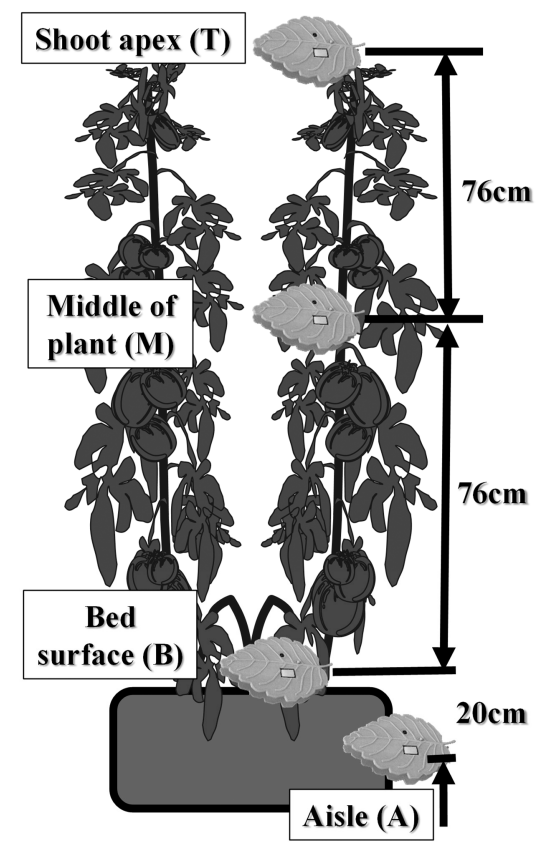

Fig. 4 Installation of "Zelko" in the experimental greenhouse and locations and labels of measurements. is given by equation (3):

$$
P E=\frac{P f r}{P r+P f r}
$$

One formula for estimating the amount in the phytochrome photoequilibrium during the light period is given by equation (4) (Smith, 1986):

$$
P E=\frac{0.75}{1+\frac{0.35 F R P F}{R P F}}
$$

On the other hand, in the dark state, Pfr slowly switches to $P r$, in a reaction that is known as the dark reversion. Assuming that the half-life of $P f r$ for dark reversion is $5 \mathrm{~h}$ (Frankland, 1986), the amount of $P E_{+5}$ after 5 min of "Zelko" measurement is given by equation (5):

$$
P E_{+5}=P E \cdot 0.5^{\frac{1}{60}}
$$

Phytochrome photoequilibrium in the plant leaf was estimated using equations (4) and (5), using the data measured by "Zelko".

\section{RESULTS AND DISCUSSION}

\section{Performance of the fabricated mobile spectrometer}

Table 1 lists the specifications of "Zelko". The device can operate continuously for more than $3 \mathrm{~d}$ using one 10 Ah mobile battery, even where electric power line is not available. Owing to its wide dynamic range, from 0.14 nmol m $\mathrm{m}^{-2} \mathrm{~s}^{-1} \mathrm{~nm}^{-1}$ to $10.67 \mu \mathrm{mol} \mathrm{m} \mathrm{m}^{-2} \mathrm{~s}^{-1} \mathrm{~nm}^{-1}$, the device can be used in a variety of illumination environments, ranging from inner areas of thick plant bushes to outdoor environments with direct sunlight.

Because a micro SD card and CSV format are utilized for recording the measured data, the data can be analyzed only using a PC that is equipped with a micro SD memory card reader and spreadsheet processing software. Figure 5 shows daily spectral photon flux measurements at position $\mathrm{T}$, acquired by "Zelko" every $5 \mathrm{~min}$; the data are presented as a $3 \mathrm{D}$ graph by the spreadsheet processing software. The dip of the Fraunhofer-A line at $\sim 760 \mathrm{~nm}$, corresponding to the absorption of gaseous $\mathrm{O}_{2}$, is clearly visible. This suggests that the device can perform spectral measurements. December 15th was a clear day. However, because the measurements were performed inside the greenhouse, a shadow that was caused by the structural material was affected, and the measured photon flux exhibited fluctuations along the temporal axis. On the other hand, Fig. 6 shows the results for the measurements that were performed during a cloudy day. The light near the shoot apex of the tomato plant was overall quite weak; furthermore, the flux of photons in the 550-700 $\mathrm{nm}$ range was further reduced. Depending on the weather, sunlight irradiation of the plant varied not only in its intensity but also in its spectral composition.

The material cost associated with the fabrication of five spectrometers was $\sim 30,000$ JPY per spectrometer, including a 10 Ah mobile battery. Because this cost is less than $1 / 10$ of the cost of a typical conventional spectrometer, we expect that continuous multi-point spectral measurements should be available in the research field of plant 
MOBILE SPECTROMETER ZELKO

Table 1 Specifications of the proposed mobile spectrometer "Zelko".

\begin{tabular}{|c|c|c|}
\hline Item & Specification & Remarks \\
\hline Size & W $171 \times$ H $36 \times$ D $100 \mathrm{~mm}^{3}$ & \\
\hline Weight & $144 \mathrm{~g}$ & Not including a mobile battery \\
\hline Supply voltage & D.C. $5 \mathrm{~V}$ & Input: USB Micro-B terminal \\
\hline Consumption current & $130 \mathrm{mAh}$ & \\
\hline Case material & Polylactic acid resin & \\
\hline Measuring wavelength & $400-800 \mathrm{~nm}$ & \\
\hline Optical resolution & $\sim 10 \mathrm{~nm}$ & From the sensor certification sheet \\
\hline Output resolution & $1 \mathrm{~nm}$ & \\
\hline Measurement interval & $5 \mathrm{~min}$ & \\
\hline Minimum sensitivity & $0.14 \mathrm{nmol} \mathrm{m}^{-2} \mathrm{~s}^{-1} \mathrm{~nm}^{-1}$ & At $532 \mathrm{~nm}$ \\
\hline Saturated photon flux & $10.67 \mu \mathrm{mol} \mathrm{m}^{-2} \mathrm{~s}^{-1} \mathrm{~nm}^{-1}$ & At $532 \mathrm{~nm}$ \\
\hline Recoding media & micro SD memory card & Up to $2 \mathrm{~GB}$ \\
\hline Output data & $\mathrm{CSV}$ format & Row: wavelength, Column: time \\
\hline
\end{tabular}

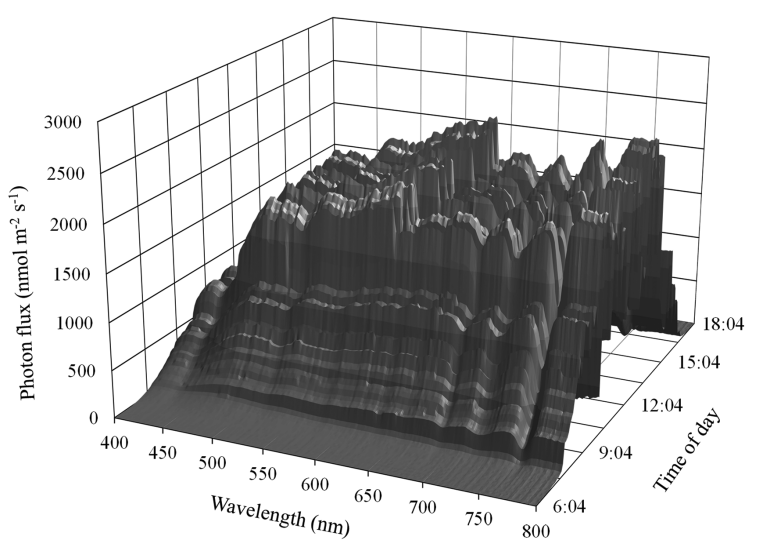

Fig. 5 Temporal dependence of the photon flux spectrum of incident light near the shoot apex of the greenhouse tomato, on December 15, 2016.

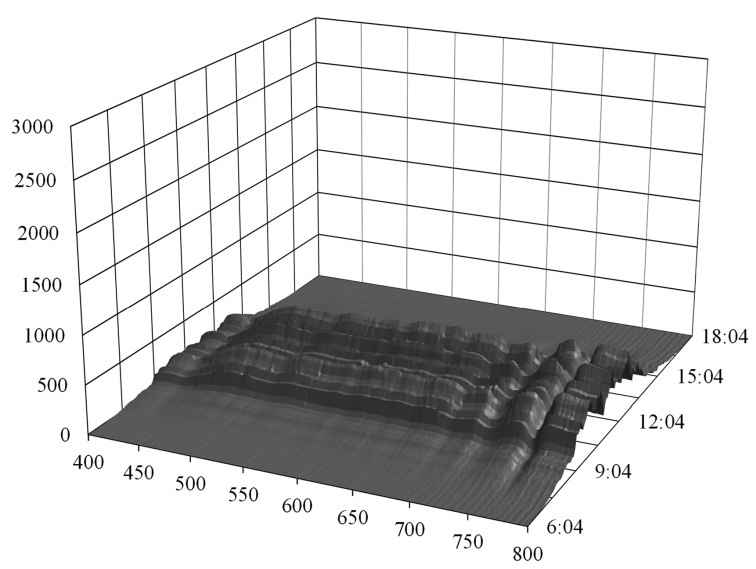

Fig. 6 Temporal dependence of the photon flux spectrum of incident light near the shoot apex of the greenhouse tomato, on December 13, 2016.

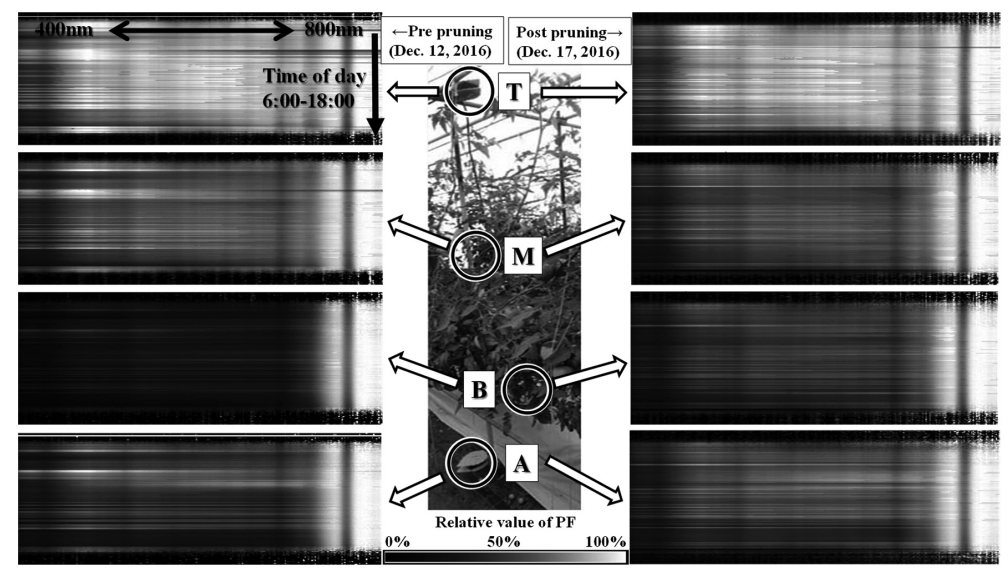

Fig. 7 Comparison of the spectroscopic light environment pre and post pruning, I terms of the maps of time of day - relative spectral photon flux.

production. Using the proposed spectrometer, plant growers will likely be able to continuously monitor the optical spectrum environments in their fields and/or greenhouses. Because farmers may find it difficult to adopt spreadsheet applications for visualization and analysis of data, we plan to incorporate a graphic liquid crystal display into "Zelko" for real-time monitoring of measurements.
Effect of pruning under leaves on irradiation environment

Figure 7 shows changes in the light environments of different measurement points, before and after pruning using gradation maps. Each gradation map (with the vertical axis representing the time of day and the horizontal axis representing wavelength) presents spectral changes in the irradiation environment during the day. Because the raw value of each $P F_{\lambda}$ changes significantly during the day, 


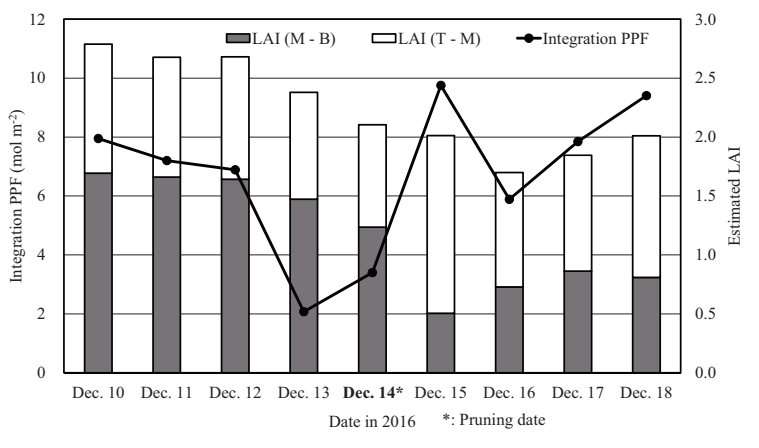

Fig. 8 Diurnal changes of estimated LAIs of greenhouse tomato plants and integration PPF near the shoot apex.

relative values, obtained by dividing each $P F_{\lambda}$ by the maximal $P F_{\lambda}$ at the time, were mapped to black and white density. The gradation map for position $\mathrm{B}$ before pruning shows that the photosynthetic effective wavelengths region (400-700 nm) was dark and the leaves of the plant were growing thick. On the other hands, after pruning the irradiation environment of position $\mathrm{B}$ was considerably improved. To quantify changes in the irradiation environment owing to pruning, the diurnal change of the estimated LAI using measured data for the far-red region was calculated and is shown in Fig. 8. Pruning was associated with a strong change in the estimated LAI between positions $M$ and $\mathrm{B}$. The mean estimated LAI between positions $\mathrm{T}$ and $\mathrm{B}$ for four $\mathrm{d}$ before and after the pruning day changed from 2.63 to 1.89 .

Distribution and temporal trends of estimated phytochrome photoequilibrium

Phytochrome plays an important role in plant morphogenesis, for example in the shade-avoidance syndrome that affect the plant's height (Morgan and Smith, 1976; Mandoli and Briggs, 1980). Transmission spectrum of greenhouse covering and shading material affect phytochrome photoequilibrium (Kittas et al., 1999). We estimated phyto- chrome photoequilibria for all tomato leaf positions, using "Zelko". Figure 9 shows temporal changes of the estimated phytochrome photoequilibrium $(P E)$ at the four positions. Regardless of the $P P F$ change, the R/FR ratio was nearly constant. Therefore, the $P E$ of the leaves near position $\mathrm{T}$ remained at $\sim 0.55$ during day. However, the fluctuations in the $P E$ at other measured positions became larger. The angle of incidence of direct sunlight changed with time, direct sunlight was often shielded by upper leaves, and the attained red light was greatly attenuated. Owing to the pruning effect, the $P E$ at position B changed its level and fluctuation in the afternoon on December 14. After pruning, direct sunlight sometimes reached at position B that was completely shielded by upper leaves. By estimating the $P E$ s for the different positions using the values measured by "Zelko", we were able to infer that the level and fluctuation of $P E$ were different at different leaf locations. We believe that the ability to measure and control optical spectrum environments in plant production fields will further improve productivity.

\section{CONCLUSION}

Applying MEMS and digital fabrication technology, a mobile spectrometer, "Zelko", was developed; the material cost of this device was $\sim 30,000$ JPY. Fabrication of "Zelko" requires very basic electronics skills. By using a greenhouse tomato plant as an experimental system, we confirmed that the fabricated device allows to simultaneously collect multipoint information on the plant's spectroscopic environment. We conclude that "Zelko" facilitates facile spectroscopic measurements for increasing plant production.

\section{ACKNOWLEDGEMENTS}

This work was supported by JSPS KAKENHI Grant Number 16K07975. We would like to express our gratitude to

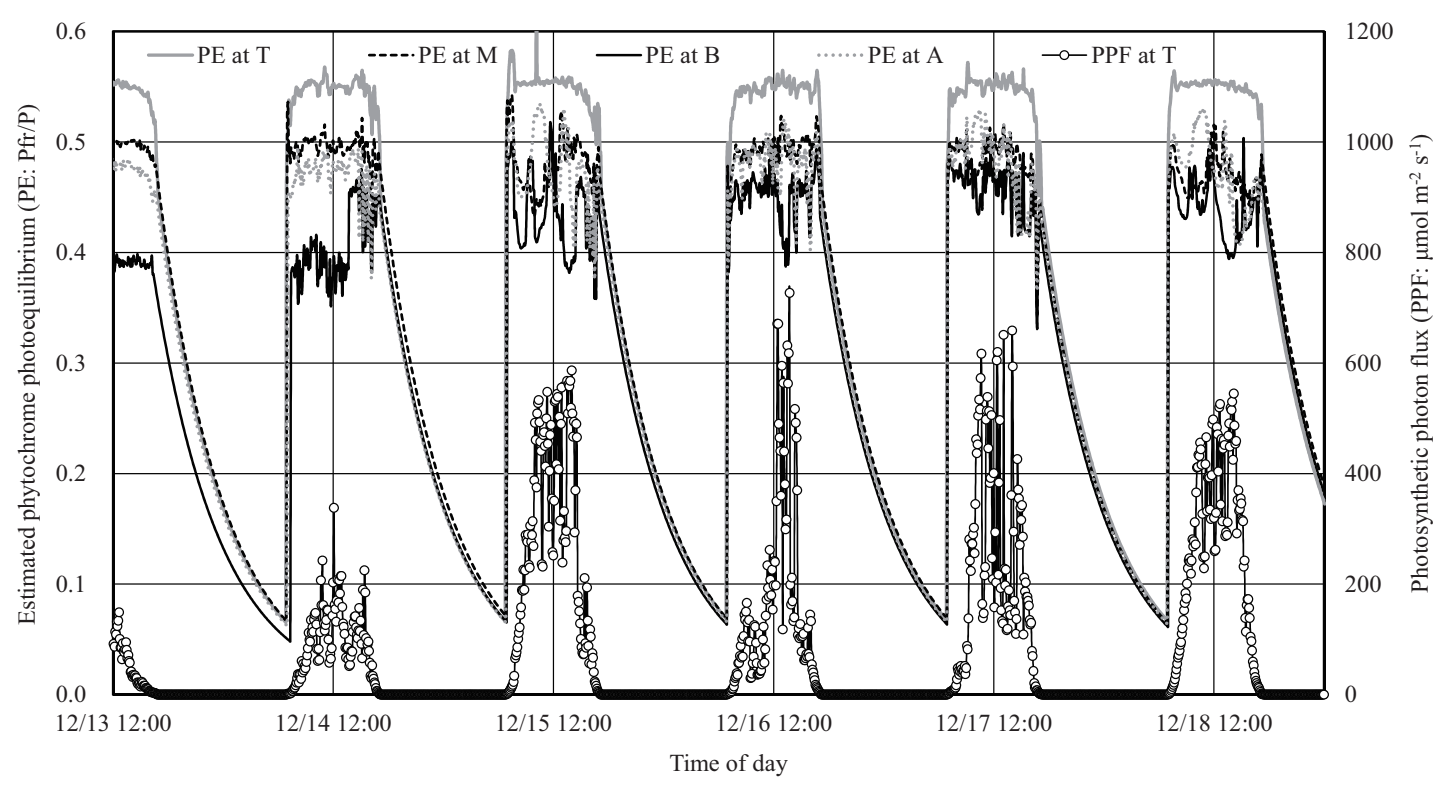

Fig. 9 Temporal changes of estimated phytochrome photoequilibrium (PE) at the four measurement points and PPF near the shoot apex. 
graduate students Mr. Kosuke Ike, Ms. Natsumi Taji, and Ms. Karin Yamaguchi, who assisted with the experiment and data processing.

\section{REFERENCES}

Frankland, B. 1986. Perception of light quantity. In "Photomorphogenesis in Plants" (ed. by Kendrick, R. E., Kronenberg, G. H. M.), Springer Science+Business Media B. V., Dordrecht, Netherlands, p 219-235.

Hamamatsu Photonics K. K. 2017. Mini-spectrometers, Hamamatsu Photonics K. K., Solid State Division, Hamamatsu, pp 44.

Holmes, M. G., Smith, H. 1975. The function of phytochrome in plants growing in the natural environment. Nature 254: 512514.

Hoshi, T., Suzuki, K., Imahara, J., Yasuba, K., Nanseki, T. 2014. Development of a nondestructive measurement system to obtain exuberance index of foliage during greenhouse crop growth by low-cost near-infrared light sensors. Acta Hortic. 1037: $627-634$.

Kaajakari, V. 2009. Practical MEMS: Design of microsystems, accelerometers, gyroscopes, RF MEMS, optical MEMS and microfluidic systems. Small Gear Publishing, Poland, pp. 496.

Kendrick, R. E., Kronenberg, G. H. M. (Eds.) 1986. Photomorphogenesis in plants. Springer Science+Business Media B. V., Dordrecht, Netherlands, pp. 580
Kittas, C., Baille, A., Giaglaras, P. 1999. Influence of covering material and shading on the spectral distribution of light in greenhouses. J. Agric. Eng. Res. 73: 341-351.

Linschitz, H., Kasche, V., Butler, W. L., Siegelman, H. W. 1966. The kinetics of phytochrome conversion. J. Biol. Chem. 241: 3395-3403.

Mandoli, D. F., Briggs, W. R. 1980. Phytochrome control of two low-irradiance responses in etiolated oat seedlings. Plant Physiol. 67: 733-739.

Morgan, D. C., Smith, H. 1976. Linear relationship between phytochrome photoequilibrium and growth in plants under simulated natural radiation. Nature 262: 210-212.

Pearce, J. M. 2012. Building research equipment with free, open-source hardware. Science 337: 1303-1304.

Smith, H. 1986. The perception of light quality. In "Photomorphogenesis in Plants" (ed. by Kendrick, R. E., Kronenberg, G. H. M.), Springer Science+Business Media B. V., Dordrecht, Netherlands, p 187-217.

Tibbitts, T. W., Morgan, D. C., Warrington, I. J. 1983. Growth of lettuce, spinach, mustard, and wheat plants under four combinations of high-pressure sodium, metal halide, and Tungsten Halogen Lamps at Equal PPFD. J. Am. Soc. Hortic. 108: 622630 .

Walter-Herrmann, J., Büching, C. (Eds.) 2014. FabLab: of machines, makers and inventors. Transcript Verlag, NordrheinWestfalen, Germany, pp 261. 
